\title{
Testing the bimodal distribution of long gamma-ray bursts in the cosmological rest-frame
}

\author{
Christian Vásconez ${ }^{1}$, Nicolás Vásquez ${ }^{1,2}$ and Ericson López ${ }^{1}$ \\ ${ }^{1}$ Quito Astronomical Observatory, Escuela Politécnica Nacional, \\ POBox 17-01-165, Quito, Ecuador \\ email: christian.vasconez@epn.edu.ec \\ ${ }^{2}$ Dept. of Physics, Escuela Politécnica Nacional, \\ Quito, Ecuador
}

\begin{abstract}
Among the several methods of classifying gamma-ray bursts (GRBs), the duration parameter has lead to the canonical classification of GRBs of long and shorts. However, the canonical classification of bursts has recently seen the emergence of a third type of GRB, which is present in a recent large burst sample from the Swift observatory. The high redshifts and the cosmological distances are directly confirmed for long bursts only, while for the short ones there is only indirect evidence for their cosmological origin. Cosmological objects should not only be redshifted in energy but also extended in time because of the expansion of the Universe. Meanwhile, an anticorrelation between the hardness and the duration is found for this subclass in contrast to the short and the long groups (Horvath et al. (2010)). Despite the differences among these three groups, it is not yet clear whether the third group represents a physically different phenomenon. In this scenario, we want to study the bimodal distribution of long bursts, focusing their temporal properties in the source location (burst frame). We have determined a temporal estimator in the cosmological rest-frame from a sample of 60 Swift's GRBs. If GRBs are at cosmological distances, then the burst profiles should be stretched in time due to cosmological time dilation by an amount proportional to the redshift, $1+z$ (Chang (2001)). Complementary, we use the hardness ratio between the soft emission (15-50 keV) and hard X-ray emission (50$150 \mathrm{keV}$ ) in order to analyze the bimodal distribution of long bursts in the time-energy plane.
\end{abstract}

Keywords. gamma ray bursts, classification

\section{Introduction}

One of the most intriguing questions is to know if the canonical classification of bursts in the observer frame is hiding some intrinsic properties due to cosmological effects (Chang (2001)). Although there has been some progress in the burst classification into three categories (Horvath et al. (2010)), classification in the burst frame are still under discussion. The analysis of the emission time (t50) (Mitrofanov et al. (1999)) and isotropic energy reveals a subclass of long GRBs when temporal estimators are cosmologically corrected. A previous analysis revealed that different time estimators from standard T90 and T50, provided a better duration of the activity of the burst engine (t50). This temporal estimator, called emission time, correlates better with the isotropic energy and shows a bimodal distribution. A subclass of dim, low redshift and longer burst in a sample of 15 GRBs simultaneously detected by Swift and Suzaku missions was determined (Vasquez and Kawai, 2010), suggesting more than one progenitor for long GRBs. To investigate whether or not this bimodality is robust, we present a complementary analysis of an extended sample of long GRBs with known $z$. 


\section{Data and analysis}

Swift bursts with known redshift are more than 200, among this data we chose a sample of long and bright GRBs, where brightness is defined as a burst with a BAT fluence at least of 30 ergs $\mathrm{cm}^{-2}$ during the period January 2006 to June 2012. There are 60 GRBs that held these characteristics but 8 where not analyzed because the lack of data or low signal to noise ratio. Then, 52 bursts were studied. We extracted spectra and light curves using Heasoft 6.11 and the analysis was done using XSPEC 12.7. The spectral model was a cut-off power low normalized to $50 \mathrm{keV}$. In order to look for spectral difference inside the sample we defined the hardness ratio $(\mathrm{HR})$ as $\frac{\left(S_{15-50}-S_{50-150}\right)}{\left(S_{15-50}+S_{50-150}\right)}$, and we plot HR as a function of $z$ in Figure 1.

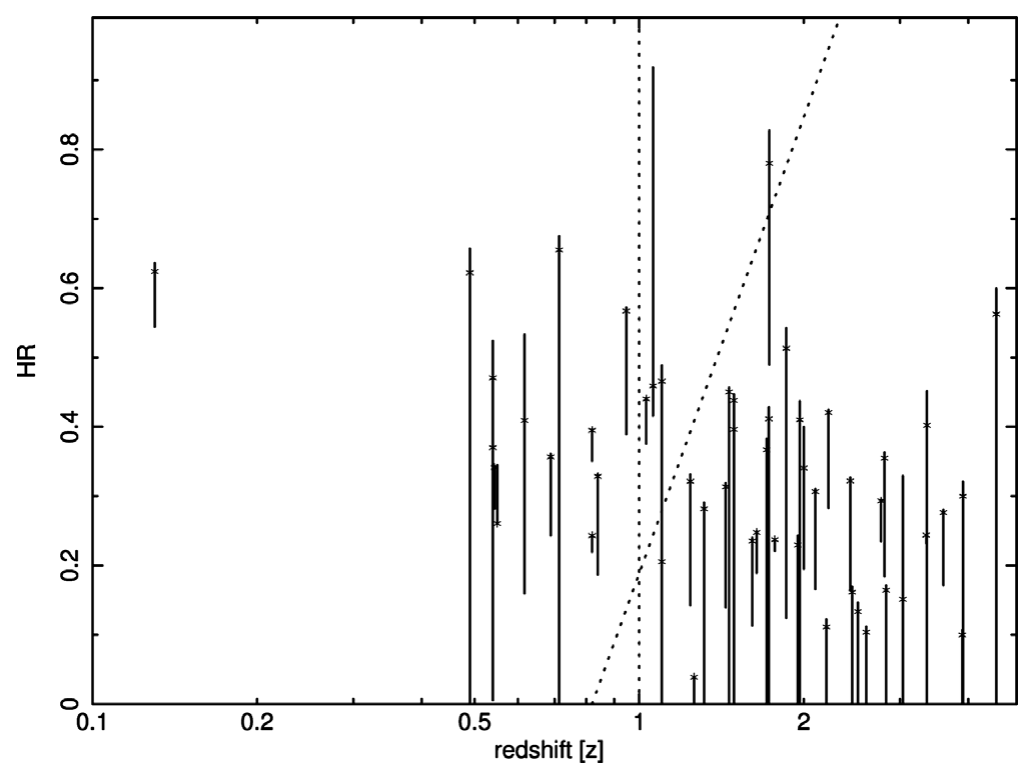

Figure 1. HR distribution as a function of $z$, suggested also a bimodal behavior. Hard burst associated with $z>1$ seems to conform a different group from those GRBs with $z<1$ that are X-ray rich.

\section{Discussion}

The evidence of a subclass of long GRBs is present in the redshift hardness ratio plane, which reinforce the possibility of a different engine or progenitor for GRBs which are X-ray rich with low redshift. The bimodality of long GRBs in the afterglow phase has been reported which is interpreted as possible difference of explosion mechanism between both types of long bursts (Dainotti et al. (2010)). In order to confirm the validity of this proposal, we are computing the emission times of the bursts in the studied sample to reproduced the bimodality in the temporal HR plane.

\section{References}

Chang H. Y. 2001, ApJL, 557, 85-88

Dainotti M. G., Willingale R., Capozziello S., Cardone V. F. \& Ostrowski M. 2010, ApJL, 722, $215-219$

Horváth I., Bagoly Z., Balázs L. G., de Ugarte Postigo A., Veres P. \& Mészáros 2010, ApJ, 713, $552-557$

Mitrofanov I., et al. 1999, ApJ, 522, 1069-1078

Page K. L., et al. 2007, ApJ, 663, 1125-1138

Vásquez N. \& Kawai N. 2010, ApJ, 713, 552-557 